\title{
The exclusion of people living with HIV (PLWH) from clinical trials in lymphoma
}

\author{
Serena Venturelli ${ }^{1}$, Alessia Dalla Pria ${ }^{2}$, Katrina Stegmann ${ }^{1}$, Paul Smith ${ }^{3}$ and Mark Bower ${ }^{\star}, 2$ \\ ${ }^{1}$ Department of HIV Medicine, Chelsea \& Westminster Hospital, London SW10 9NH, UK; ${ }^{2}$ Department of Oncology, National Centre \\ for HIV Malignancies, Chelsea \& Westminster Hospital, London SW10 9NH, UK and ${ }^{3}$ Tumour Group Lead for Haematology and Brain \\ Trials, Cancer Research UK and UCL Cancer Trials Centre, 90 Tottenham Court Road, London W1T 4TJ, UK
}

Background: The prognosis of non-Hodgkin lymphoma and Hodgkin lymphoma is not affected by HIV serostatus, yet people living with HIV (PLWH) are frequently excluded from clinical trials in lymphoma.

Methods: The UK NIHR Clinical Research Network Study Portfolio website was used to identify all the open clinical trials in lymphoma in the United Kingdom in January 2015. Trials that excluded PLWH were further investigated to evaluate if the exclusion was justified by scientific evidence.

Results: We identified 56 multicentre open clinical trials in lymphoma including 46 interventional trials. People living with HIV were excluded from 32 interventional trials (70\%). We identified a biologically valid reason (a potential increased risk of greater immunosuppression) for excluding PLWH from one trial and possibly for one optional arm in another study.

Conclusions: There was no scientific or safety justification for excluding PLWH from most lymphoma clinical trials included on the NIHR portfolio. A clear justification for excluding PLWH was not offered in the available protocols. The exclusion of PLWH should be explicitly justified on scientific grounds in protocols to minimise stigmatisation.

People living with HIV (PLWH) are at an increased risk of both high-grade non-Hodgkin lymphoma (NHL) and Hodgkin lymphoma (HL) (Cote et al, 1997; Petruckevitch et al, 1999; Herida et al, 2003; Engels et al, 2008). Over the past two decades following the introduction of combination antiretroviral therapy, the prognosis of NHL and HL in PLWH has dramatically improved with survival now equal to that observed in the HIV negative population (Montoto et al, 2012; Coutinho et al, 2014).

The British HIV Association evidence-based Guidelines for the management of HIV-associated malignancies published in 2014 are accredited by National Institute for Health and Care Excellence. They recommend the treatment of PLWH and NHL/HL with the same chemotherapy protocols used in the general population (Bower et al, 2014).

In 2006 the US National Cancer Institute stated that 'individuals known to be HIV-positive should not be arbitrarily excluded from participation in clinical cancer treatment trials' and that a scientific justification needs to be provided in case they are excluded (National
Cancer Institute, Cancer Therapy Evaluation Program, 2006). However a study of lung cancer clinical trials in the United States in 2008 demonstrated that PLWH were excluded with little or no scientific justification (Persad et al, 2008). No other publications have addressed the eligibility of PLWH for cancer trials.

The National Institute for Health Research (NIHR) is a UK government body that co-ordinates and funds research that was established in 2006. Between 2006 and $2012>3$ million patients were recruited to NIHR accredited high-quality clinical research studies. Clinical trials are evaluated for scientific value and rigour before being included on the NIHR portfolio of studies, which then attracts NHS support for research infrastructure. Amongst the aims of NIHR are to 'increase the opportunities for patients and the public to participate in, and benefit from, research' and to 'promote and protect the interests of patients and the public in health research'. The portfolio of trials supported by NIHR includes trials funded by industry charities, governments, as well as investigator initiated commercial collaborative research. 
We believe that PLWH may benefit from clinical trials but are excluded from recruitment on account of exclusion criteria, including clinical trials for lymphoma. In some cases the exclusion of PLWH from clinical trials will be based on valid scientific reasoning, for example the use of live vaccines or profoundly immunosuppressive therapies. In other circumstances, potential pharmacokinetic drug interactions may require the exclusion of PLWH who are receiving antiretrovirals that could interact with experimental trial drugs. We examined the exclusion criteria for all currently recruiting NIHR accredited and supported lymphoma trials to assess whether PLWH were excluded from recruitment and whether this exclusion was scientifically justified.

\section{AIM AND OBJECTIVES}

The overall aim of our study was to review the open clinical trials for lymphoma in the UK that are included on the NIHR portfolio and to evaluate if the exclusion of PLWH was justified by scientific evidence.

To achieve this aim the following objectives were set:

1. Evaluate whether PLWH were excluded from clinical trials in lymphoma in UK.

2. Evaluate whether a clear justification for exclusion was offered in the protocols.

3. Assess if increased risk of immunodeficiency could justify the exclusion of PLWH.

4. Assess if pharmacokinetic interactions could justify the exclusion of PLWH.

\section{MATERIALS AND METHODS}

The UK NIHR Clinical Research Network Study Portfolio website was used to identify all the open clinical trials in lymphoma in the United Kingdom in January 2015 and their inclusion and exclusion criteria. Protocols, product information sheets and published data were used to evaluate mechanism of action, toxicity, metabolism and pharmacokinetic interactions of novel or only recently licensed drugs.

\section{RESULTS}

We identified 56 multicentre open clinical trials in lymphoma. Ten were exclusively observational trials, one was an interventional and observational trial, and 45 were exclusively interventional trials. We investigated the inclusion and exclusion criteria of the 46 interventional trials.

PLWH were eligible for 13 out of 46 trials and if the plasma HIV viral load was fully suppressed for one further trial. PLWH were excluded from the remaining 32 trials $(70 \%)$. The review of the screening visit mandatory tests of the 32 trials excluding PLWH showed that in $55 \%$ of the trials for which this information was available, an HIV test was not required. In these cases the exclusion criteria was based on known history of HIV seropositive status, therefore people with undiagnosed HIV infection were potentially put at risk, assuming that the exclusion criteria was justified.

Of the 32 trials excluding PLWH, 2 trials $(6 \%)$ were phase I, 10 trials $(31 \%)$ were phase II, 1 trial (3\%) was phase II/III, 18 trials (56\%) were phase III and 1 trial (3\%) was phase IV. The trials included novel drugs/treatment approaches or licensed drugs in an unlicensed indication. Eight different classes of drugs were identified: monoclonal antibodies (13 trials), cytotoxic agents (5 trials), Bruton tyrosine kinase inhibitors (4 trials), proteasome inhibitors (3 trials), Phosphoinositide 3-kinase inhibitors (2 trials), serine/threonine protein kinase inhibitors (1 trial), Bcl-2 inhibitors (1 trial) and monocarboxylate transporter inhibitors (1 trial). The efficacy of herpes zoster vaccine was assessed in one trial and the efficacy of surgery alone in another trial.

Complete trial protocols were requested for the 32 trials excluding PLWH but were only provided by 9 trials (28\%). In no case was the exclusion of PLWH explicitly justified in the protocol. The trial management teams were contacted for the remaining 23 trials. Four (13\%) responded to specific questions, stating they had not specifically outlined the reason for excluding HIV patients in their studies. Only 1 team declared that the reason was 'to mitigate against any safety risks for these patients through risk of infection'. For the remaining 19 trials (59\%) the management teams declared that the protocols were 'confidential commercial documents only available to investigators participating in the studies' and therefore it was not possible to verify if a scientific reason to exclude PLWH was offered.

We reviewed the potential increased risk of toxicity related to immunosuppression for HIV patients in the 32 trials excluding PLWH and we identified a biologically valid reason for excluding PLWH for one trial and a possible valid reason for one further study. In the first case the study was a Phase I trial on a monocarboxylate transporter 1 inhibitor known to be a potent immunosuppressor which blocks T-lymphocyte proliferation (Murray et al, 2005). In the second case the trial was investigating a serine/threonine protein kinase inhibitor drug (alisertib) vs an investigator choice which included romidepsin. Romidepsin is a histone deacetylase inhibitor that has been demonstrated to be a potent activator of latent HIV ex vivo (Wei et al, 2014). However, in this trial the investigator had the possibility to choose between two other options (pralatrexate and gemcitabine) with no risk of increased toxicity for PLWH and could have exercised this choice for PLWH. No increased risk of toxicity was identified in the other 30 (94\%) trials excluding PLWH.

In addition, we reviewed the potential pharmacokinetic interaction between antiretroviral agents (ARVs) and the investigational agents or the compared standard chemotherapeutic agents. Potential pharmacokinetic interactions were identified in 28 out of 32 trials $(87 \%)$. The majority of chemotherapeutic agents included in the clinical trial protocols are metabolised by the hepatic cytochromes $\mathrm{P} 450$ enzymes including the isoforms CYP2B6, CYP2C19 and CYP3A4/5. Both non-nucleoside reverse-transcriptase inhibitors (NNRTIs) and boosted protease inhibitors (PIs) classes of antiretrovirals can act as inducers or inhibitors of these CYP enzymes, potentially altering the pharmacokinetics of the chemotherapeutic agents. However, there are antiretroviral drugs such as the majority of nucleoside reverse transcriptase inhibitors (NRTIs) and some integrase inhibitors (II) that do not alter CYP enzyme activity. In most PLWH who develop lymphomas, ARVs are switched to NRTI and II (viral resistance profile permitting) to avoid these drug interactions with chemotherapy. If a similar switch were allowed in the lymphoma clinical trials, PLWH could be eligible for these trials.

Out of the four trials (12\%) with no risk of pharmacokinetic interaction, two were phase I studies investigating a monocarboxylate transporter drug and an anti-CD19 monoclonal antibody drug, one was a trial investigating the efficacy of Rituximab plus Lenalidomide $v s$ Rituximab in relapsed/refractory indolent NHL and one was a trial on the efficacy of Herpes Zoster vaccine in haematopoietic stem cell transplant recipients.

\section{CONCLUSION}

There was no scientific or safety justification for excluding PLWH from most lymphoma clinical trials included on the NIHR 
portfolio. A clear justification for excluding PLWH was not offered in the available protocols. Increased risk of immunosuppression toxicity by anti-lymphoma agents were found in only $6 \%$ of the trials. The selection of antiretroviral drugs avoiding NNRTI and PIs would have enabled eligibility with no risk of drug interactions.

Our study shows that PLWH could potentially access a large number of lymphoma trials with personal and scientific medical benefit. Protocols authors should be encouraged to explain the reasons behind the exclusion of PLWH to guarantee fair access to clinical trials for PLWH.

\section{ACKNOWLEDGEMENTS}

SV, PS and MB designed the research study, SV and PS collected the data, SV, ADP, KS and MB analysed the data, SV, ADP, KS, PS and $\mathrm{MB}$ wrote the paper.

\section{CONFLICT OF INTEREST}

The authors declare no conflict of interest.

\section{REFERENCES}

Bower M, Palfreeman A, Alfa-Wali M, Bunker C, Burns F, Churchill D, Collins S, Cwynarski K, Edwards S, Fields P, Fife K, Gallop-Evans E, Kassam S, Kulasegaram R, Lacey C, Marcus R, Montoto S, Nelson M, Newsom-Davis T, Orkin C, Shaw K, Tenant-Flowers M, Webb A, Westwell S, Williams M (2014) British HIV Association guidelines for HIV-associated malignancies 2014. HIV Med 15(Suppl 2): 1-92.

Cote TR, Biggar RJ, Rosenberg PS, Devesa SS, Percy C, Yellin FJ, Lemp G, Hardy C, Geodert JJ, Blattner WA (1997) Non-Hodgkin's lymphoma among people with AIDS: incidence, presentation and public health burden. AIDS/Cancer Study Group. Int J Cancer 73(5): 645-650.

Coutinho R, Pria AD, Gandhi S, Bailey K, Fields P, Cwynarski K, Wilson A, Papanastasopoulos P, Tenant-Flowers M, Webb A, Burns F, Marcus RE,
Orkin C, Montoto S, Bower M (2014) HIV status does not impair the outcome of patients diagnosed with diffuse large B-cell lymphoma treated with R-CHOP in the cART era. AIDS 28(5): 689-697.

Engels EA, Biggar RJ, Hall HI, Cross H, Crutchfield A, Finch JL, Grigg R, Hylton T, Pawlish KS, McNeel TS, Goedert JJ (2008) Cancer risk in people infected with human immunodeficiency virus in the United States. Int $J$ Cancer 123(1): 187-194.

Herida M, Mary-Krause M, Kaphan R, Cadranel J, Poizot-Martin I, Rabaud C, Plaisance N, Tissot-Dupont H, Boue F, Lang JM, Costagliola D (2003) Incidence of non-AIDS-defining cancers before and during the highly active antiretroviral therapy era in a cohort of human immunodeficiency virus-infected patients. J Clin Oncol 21(18): 3447-3453.

Montoto S, Shaw K, Okosun J, Gandhi S, Fields P, Wilson A, Shanyinde M, Cwynarski K, Marcus R, de Vos J, Young AM, Tenant-Flowers M, Orkin C, Johnson M, Chilton D, Gribben JG, Bower M (2012) HIV status does not influence outcome in patients with classical hodgkin lymphoma treated with chemotherapy using doxorubicin, bleomycin, vinblastine, and dacarbazine in the highly active antiretroviral therapy era. J Clin Oncol 30(33): 4111-4116.

Murray CM, Hutchinson R, Bantick JR, Belfield GP, Benjamin AD, Brazma D, Bundick RV, Cook ID, Craggs RI, Edwards S, Evans LR, Harrison R, Holness E, Jackson AP, Jackson CG, Kingston LP, Perry MW, Ross AR, Rugman PA, Sidhu SS, Sullivan M, Taylor-Fishwick DA, Walker PC, Whitehead YM, Wilkinson DJ, Wright A, Donald DK (2005) Monocarboxylate transporter MCT1 is a target for immunosuppression. Nat Chem Biol 1(7): 371-376.

National Cancer Institute, Cancer Therapy Evaluation Program (2006) Guidelines regarding the inclusion of cancer survivors and HIV-positive individuals on clinical trials. Institute NC. Bethesda, MD, USA.

Persad GC, Little RF, Grady C (2008) Including persons with HIV infection in cancer clinical trials. J Clin Oncol 26(7): 1027-1032.

Petruckevitch A, Del Amo J, Phillips AN, Stephenson JM, Johnson AM, De Cock KM (1999) Risk of cancer in patients with HIV disease. London African HIV/AIDS Study Group. Int J STD AIDS 10(1): 38-42.

Wei DG, Chiang V, Fyne E, Balakrishnan M, Barnes T, Graupe M, Hesselgesser J, Irrinki A, Murry JP, Stepan G, Stray KM, Tsai A, Yu H, Spindler J, Kearney M, Spina CA, McMahon D, Lalezari J, Sloan D, Mellors J, Geleziunas R, Cihlar T (2014) Histone deacetylase inhibitor romidepsin induces HIV expression in CD4 T cells from patients on suppressive antiretroviral therapy at concentrations achieved by clinical dosing. PLoS Pathog 10(4): e1004071. 